\title{
ARTICLE
}

Molecular Diagnostics

\section{Retinoid X receptor gamma (RXRG) is an independent prognostic biomarker in ER-positive invasive breast cancer}

\author{
Chitra Joseph ${ }^{1}$, Sara Al-Izzi ${ }^{1}$, Mansour Alsaleem ${ }^{1}$, Sasagu Kurozumi ${ }^{1}$, Michael S Toss ${ }^{1,2}$, Maariya Arshad ${ }^{1}$, Fang Qin Goh ${ }^{1}$, \\ Ibraheem M. Alshankyty ${ }^{3}$, Mohammed A. Aleskandarany ${ }^{1,2}$, Simak Ali ${ }^{4}$, lan O. Ellis ${ }^{1}$, Nigel P. Mongan ${ }^{5,6}$, Andrew R. Green $\mathbb{D}^{1}$ and \\ Emad A. Rakha ${ }^{1,2}$
}

BACKGROUND: Retinoid X Receptor Gamma (RXRG) is a member of the nuclear receptor superfamily and plays a role in tumour suppression. This study aims to explore the prognostic significance of RXRG in breast cancer.

METHODS: Primary breast cancer tissue microarrays $(n=923)$ were immuno-stained for RXRG protein and correlated with clinicopathological features, and patient outcome.

RESULTS: Nuclear RXRG expression was significantly associated with smaller tumour size $(p=0.036)$, lower grade $(p<0.001)$, lobular histology $(p=0.016)$, lower Nottingham Prognostic Index $(p=0.04)$ and longer breast cancer-specific survival $(p<0.001)$, and longer time to distant metastasis $(p=0.002)$. RXRG expression showed positive association with oestrogen receptor (ER)related biomarkers: GATA3, FOXA1, STAT3 and MED7 (all $p<0.001$ ) and a negative correlation with the Ki67 proliferation marker. Multivariate analysis demonstrated RXRG protein as an independent predictor of longer breast cancer-specific survival and distant metastasis-free survival. In the external validation cohorts, RXRG expression was associated with improved patients' outcome $(p=$ 0.025). In ER-positive tumours, high expression of RXRG was associated with better patient outcome regardless of adjuvant systemic therapy. ER signalling pathway was the top predicted master regulator of RXRG protein expression $(p=0.005)$.

CONCLUSION: This study provides evidence for the prognostic value of RXRG in breast cancer particularly the ER-positive tumours.

British Journal of Cancer (2019) 121:776-785; https://doi.org/10.1038/s41416-019-0589-0

\section{INTRODUCTION}

Breast cancer is the most common cancer among women worldwide. ${ }^{1}$ Oestrogen receptor (ER) and progesterone receptor $(\mathrm{PR})$, which are members of the nuclear receptor superfamily of transcription factors, are important in predicting prognosis and establishing therapeutic strategies for breast cancer treatment. Recent studies have revealed growing evidence of the involvement of nuclear receptors, other than ER and PR, in breast cancer development and progression. ${ }^{2,3}$ Drugs targeting nuclear receptors are widely used in the clinic for treating patients. ${ }^{4}$ Expression levels of some nuclear receptors, such as thyroid hormone receptor beta (THRb), COUP transcription factor 2 (COUP-TF2), peroxisome proliferator-activated receptor gamma (PPARG) and liver receptor homologue-1 (LRH-1), are associated with clinicopathological variables and can predict outcome in tamoxifentreated patients. ${ }^{5}$ The glucocorticoid receptor (GR) in breast cancer exerts anti-proliferative and anti-apoptotic activities and its overexpression is associated with features characteristic of longer survival. ${ }^{6,7}$ Moreover, in tamoxifen-treated ER-positive breast cancer, androgen receptor (AR; also a member of the nuclear receptor superfamily) status has prognostic value and it is reported to be a crucial factor in deciding treatment regime. ${ }^{8}$
With these important roles in breast cancer, other nuclear receptors could therefore provide additional therapeutic targets for breast cancer management. ${ }^{9-11}$

Retinoids derived from vitamin A are signalling molecules that play important roles in cell differentiation and proliferation ${ }^{12}$ and act via retinoic acid receptors (RARs) and retinoid $X$ receptors (RXRs), which are members of the nuclear receptors superfamily. Retinoids are well documented for their ability to induce differentiation and arrest proliferation in cancer. ${ }^{12,13}$ The RXR family are known to form heterodimers with other nuclear receptors, including the vitamin D receptor (VDR), peroxisome proliferator-activated receptors (PPARs) and RARs. ${ }^{11}$ There are three subtypes of the Retinoid X Receptor (RXR), namely RXR Alpha (RXRa; NR2B1), RXR $\beta$ (NR2B2) and RXRY (NR2B3). ${ }^{14}$ These receptors have tumour suppressor properties, particularly as their ligand 9-cis-retinoic acid, ${ }^{12}$ and impede cellular proliferation. ${ }^{15}$ Moreover, the RXR family are involved in mediating the antiproliferative effects of retinoic acid (RA) as partners of RARB and RARA. ${ }^{12}$

RXRG has been demonstrated to modulate cellular differentiation and apoptosis in different tumour types. For example, enhanced expression of RXRG was associated with increased

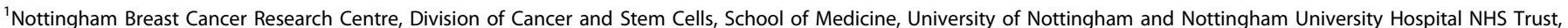

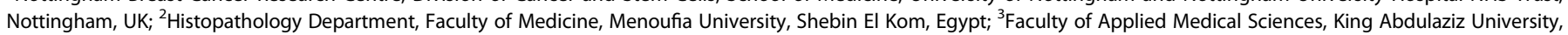

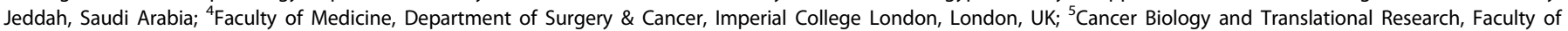
Medicine and Health Sciences, University of Nottingham, Nottingham, UK and ${ }^{6}$ Department of Pharmacology, Weill Cornell Medicine, New York, NY 10065, USA Correspondence: Emad A. Rakha (Emad.Rakha@nottingham.ac.uk)
}

Received: 11 April 2019 Revised: 22 August 2019 Accepted: 5 September 2019

Published online: 27 September 2019 
apoptosis in ovarian cancer. ${ }^{16}$ Epigenetic silencing of RXRG correlated with decreased overall survival in lung cancer. ${ }^{17}$ In ovarian cancer tumour models, RXRG activation re-sensitises ovarian carcinoma cells to apoptosis. However, the mechanism by which this occurs is still unclear. With minimal toxicity both in vitro and in vivo, novel RXR family members (rexinoids), have been reported to suppress breast cancer development in several animal models and have been extensively evaluated either alone or in combination with selective ER modulators. ${ }^{18}$ One RXRG partner, RARA was shown to influence the ERa transcriptional complex in oestrogen-treated MCF-7 breast cancer cells. ${ }^{19,20}$ Altogether, these findings indicate that RXRG could have a function in tumour pathogenesis and could potentially be promising cancer therapeutics.

Therefore, this study aimed to investigate the potential prognostic role of RXRG in breast cancer with a focus on the luminal ER-positive class.

\section{METHODS}

Study cohort

This study was conducted on a large cohort $(n=923)$ of primary breast cancer from patients who presented to Nottingham City Hospital with available clinicopathological data, as previously described. ${ }^{21-23}$ Treatment and outcome data, including breast cancer-specific survival and distant metastasis-free interval was maintained on a prospective basis. Breast cancer-specific survival was defined as the duration (in months) from the date of primary surgery to the time of death because of breast cancer. Distant metastasis-free interval was defined as the duration (in months) from primary surgical treatment to the occurrence of first distant recurrence.

Evaluation of RXRG protein expression RXRG protein expression was evaluated using immunohistochemistry (IHC) preceded by validation of the rabbit RXRG antibody (Abcam, ab15518) specificity using western blot. For the latter, cell lysates of MDA-MB-231 and MCF-7 cell lines (obtained from the
American Type Culture Collection; Rockville, MD, USA) were incubated with the primary antibody at 1:700 dilution. The specificity of the antibody was validated with a single specific band at the predicted molecular weight ( $39 \mathrm{kDa}$, Fig. 1a).

For evaluation of the morphological pattern of protein expression and suitability of tissue microarrays for its assay, immunohistochemistry was assessed in full-face breast cancer tissue sections $(n=10)$. Tumour samples were arrayed onto tissue microarrays as previously described. ${ }^{21}$ Four micrometre sections from the tissue microarrays and full-face sections were immunohistochemically stained using the Novolink Max Polymer Detection system (Leica, Newcastle, UK). The antibody was incubated $24 \mathrm{~h}$ at the concentration of 1:300.

The modified Histo-score ( $H$-score) method was used in assessing immunohistochemistry staining, taking the staining intensity and percentage positivity into account. ${ }^{24}$ High-resolution digital images were generated via scanning the stained slides using Nanozoomer (Hamamatsu Photonics, Welwyn Garden City, UK) at $\times 20$ magnification to facilitate the scoring of the tissue microarrays cores. The sections were blindly double scored by two researchers, including a consultant histopathologist for $\sim 25 \%$ cores to assess inter-observer concordance. Inter-observer agreement was determined, and the intra-class correlation co-efficient was $\mathbf{0 . 8 3}$, indicating an excellent concordance between scorers. Moreover, the discordant cases were re-scored by the both observers and a consensus score was agreed and assigned. Biomarkers closely relevant to breast cancer carcinogenesis, progression and outcome were also available for this cohort of patients (See Tables 2 and 3). Immunohistochemistry staining and dichotomisation of these biomarkers were used as per previous publications. $^{6,22,25-33}$

\section{Gene expression cohorts}

The clinicopathological significance of RXRG mRNA expression was assessed using a subset $(n=150)$ of the Nottingham series that was included in the Molecular Taxonomy of Breast Cancer International Consortium (METABRIC) data set. ${ }^{34}$ The aim of this investigation is to understand the molecular biology of RXRG
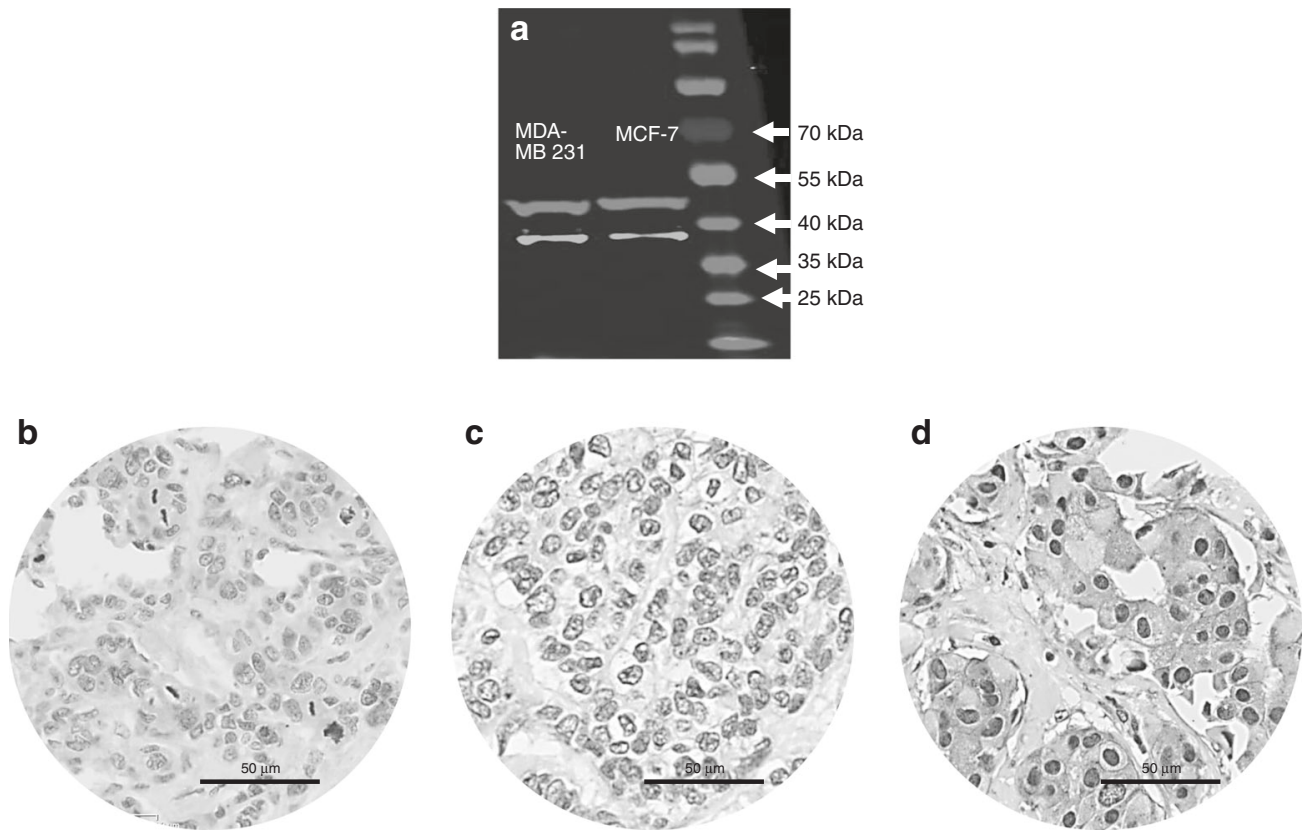

Fig. 1 Western blot and immunohistochemical expression of RXRG in breast cancer. a Western blotting results for RXRG expression in MCF-7 and MDA-MB231 breast cancer cell lines using rabbit polyclonal antibody (Abcam, ab15518). Green and red bands represent RXRG and the house-keeping Beta-Actin, respectively. RXRG protein expression in breast cancer tissue microarrays cores. b Negative/no staining c showing low expression and $\mathbf{d}$ showing high immunoreactivity. Images are at $\mathrm{x} 40$ magnification 
protein expression as an end product, therefore, the analysis was completed utilising cases with RXRG protein expression. The definition of cases into low versus high groups was based on RXRG protein expression.

External validation was performed using the Breast Cancer Gene-Expression Miner v4.0 (bc-GenExMiner v4.0), ${ }^{35}$ as previously described. ${ }^{33,36}$ Breast cancer cases data set $(n=818)$ within The Cancer Genome Atlas (TCGA) ${ }^{37}$ was also used for external validation of RXRG mRNA expression. Patient outcome following systemic treatment was further validated using KM Plotter $(n=$ 3951). ${ }^{38}$

\section{Pathway analysis}

Differential gene expression between RXRG negative and positive cases was assessed using the Robina implementation of EdgeR. ${ }^{39}$ Differential expression with $>2$-fold difference and a false discovery rate of $q<0.05$ between $R X R G$-negative and -positive cases were considered significant. Webgestalt (http://www. webgestalt.org) was used to annotate the differential gene expression list and to identify over-represented gene ontologies and pathways. ${ }^{40}$

Statistical analysis

IBM SPSS 22.0 (Chicago, IL, USA) software was used for statistical analysis. The $\mathrm{H}$-scores of expressions of nuclear RXRG did not follow a normal distribution. For this reason, expression of RXRG protein was used to define patient groups based on prediction of breast cancer-specific survival using X-tile (http://tissuearray.org; Yale University, USA). ${ }^{41}$ Chi-squared test was used to evaluate the association between expression of other biomarkers and the clinicopathological parameters. Correlation between RXRG and ER/PR expression was analysed using Spearman's correlation co-efficient test. Association between RXRG expression, clinicopathological parameters and other related biomarkers using the continuous $\mathrm{H}$-score were evaluated.

Kaplan-Meier analysis with log-rank test for significance was performed to assess breast cancer-specific survival and distant metastasis-free interval. Interaction between RXRG and ER was evaluated using Cox regression model, which was also used for multivariate survival analysis with adjustment of covariates to test independence from standard prognostic factors in breast cancer (nodal stage, tumour grade, tumour size, ER level of expression (defined as percentage of positive tumour cells), and Ki67. The STRING database (http://string-db.org) ${ }^{42}$ was used to evaluate the interaction with RXRG and other nuclear receptors in steroid signalling pathways. The $p$-values were adjusted using Bonferroni correction for multiple testing. A $p$-value of $<0.05$ was considered significant.

This study obtained ethics approval by the North West-Greater Manchester Central Research Ethics Committee under the title: Nottingham Health Science Biobank (NHSB), reference number $15 / \mathrm{NW} / 0685$. All samples from Nottingham used in this study were pseudo-anonymised and collected prior to 2006 and stored in compliance with the UK Human Tissue Act.

\section{RESULTS}

RXRG protein expression

Full-face tissue sections (Supplementary Fig. 1a-c) showed high RXRG expression in the normal glandular epithelium (Supplementary Fig. 1b). In contrast, low RXRG immunopositivity was observed in the nuclei of invasive cancer cells (Supplementary Fig. 1c), with some malignant cells additionally featuring cytoplasmic staining. On tissue microarrays, RXRG protein expression levels varied from absent to high (Fig. 1b-d). In the 923 scorable cores, the cutoff points of the RXRG nuclear $\mathrm{H}$-score was set at 175 by X-tile analysis, where low expression is defined as $\mathrm{H}$-scores $<175$ and high expression as $H$-scores $\geq 175$. Low RXRG nuclear expression was observed in 458/923 (49.6\%) cases and high expression was observed in 465/923 (50.4\%) cases. Low RXRG mRNA expression was found in $73 / 150$ (49\%), whereas high RXRG mRNA expression was observed in $77 / 150(51 \%)$ cases.

\section{Relationship between RXRG protein expression and} clinicopathological variables

In the whole cohort and ER-positive sub-cohort, RXRG was associated with features of favourable prognosis, including smaller tumour size $(p=0.036)$, lower histological grade $(p<0.00001)$, less pleomorphism $(p=0.042)$, lower mitotic scores $(p<0.00001)$, lobular and special tumour types of excellent prognosis $(p=$ $0.016)$, and lower Nottingham Prognostic Index ( $p<0.05$; Table 1$)$. Moreover, significant association was observed with breast cancer molecular intrinsic subtypes $(p<0.00001$ and $p=0.009)$, for the whole series and ER-positive tumours, respectively (Table 1). High RXRG expression was primarily observed in luminal $A$ tumours (136/214, 63.6\%), while it was less expressed in HER2 + and triplenegative breast cancer.

There was a significant positive linear correlation between RXRG and ER expression in the whole cohort and in ER-positive tumours ( $r=0.30, p<0.0001$ and $r=0.20, p=0.016$, respectively). Similar results were observed with PR expression $(r=0.20, p=0.014$ and $r=0.17, p=0.016$; respectively). High-nuclear RXRG expression showed significant positive association with ER and PR positivity ( $p<0.0001$ and $p=0.018$, respectively), while negative association was observed with basal cytokeratin CK5/6 ( $p=0.020$; Table 2). High expression of RXRG was positively associated with luminal subtype-related biomarkers in both the whole cohort and ERpositive tumours, including ER-chromatin interaction regulator Forkhead box protein A1 (FOXA1; $p<0.00001)$ and human brainexpressed X-linked1 (BEX1; $p<0.00001)$. Significant positive associations were observed with cell cycle regulatory proteins such as GATA3 $(p=0.0001)$, and STAT3 $(p<0.00001)$; markers also known to be over-expressed in ER-positive breast cancer and associated with favourable outcome. ${ }^{21,43}$ By contrast, negative associations were observed with the proliferation marker Ki67 ( $p=0.014)$, epithelial-mesenchymal transition markers such as $\mathrm{N}$ cadherin $(p<0.00001)$ and phosphatidylinositol-4,5-Bisphosphate 3-Kinase Catalytic Subunit Alpha (PIK3CA; $p=0.012$ ). In addition, the mediator subunit MED7 was positively associated $(p<0.00001)$ with RXRG (Table 2; both whole and ER-positive cohort). In ERnegative tumours, only MED7 $(p<0.00001), \operatorname{BEX} 1(p=0.032)$ and $\mathrm{N}$-cadherin $(p=0.034)$ showed significant association with RXRG (Table 2).

Positive associations were observed between the nuclear expression of RXRG and the expression of nuclear receptors, including PPARY, PPAR $\beta, A R, R A R a$, glucocorticoid receptor and liver receptor homologue-1 ( $p$ for all $<0.001$ ) (Table 3; in the whole cohort, ER-positive and ER-negative cohort). Moreover, using the continuous $\mathrm{H}$-score to assess the association between RXRG expression and the clinicopathological parameters, as well as other breast cancer-related biomarkers revealed similar significant association to those obtained with the categorised RXRG (Supplementary Table 1).

\section{Association between RXRG protein expression and patients' outcome}

High expression of RXRG was associated with longer breast cancer-specific survival ( $p<0.0001$; Fig. 2a). Regarding distant metastasis, high RXRG expression was associated with a lower probability of distant metastasis ( $p=0.002$; Fig. $2 \mathrm{~b}$ ). Cox proportional multivariate analysis showed that RXRG expression was an independent indicator of both longer breast cancer-specific survival and distant metastasis-free interval in the whole cohort $(\mathrm{HR}=0.6 ; 95 \% \mathrm{Cl}=0.4-0.8 ; p=0.04$ and $\mathrm{HR}=0.7 ; 95 \% \mathrm{Cl}=$ $0.6-0.9 ; p=0.025$, respectively) independent of the standard prognostic parameters of breast cancer, including tumour size, 


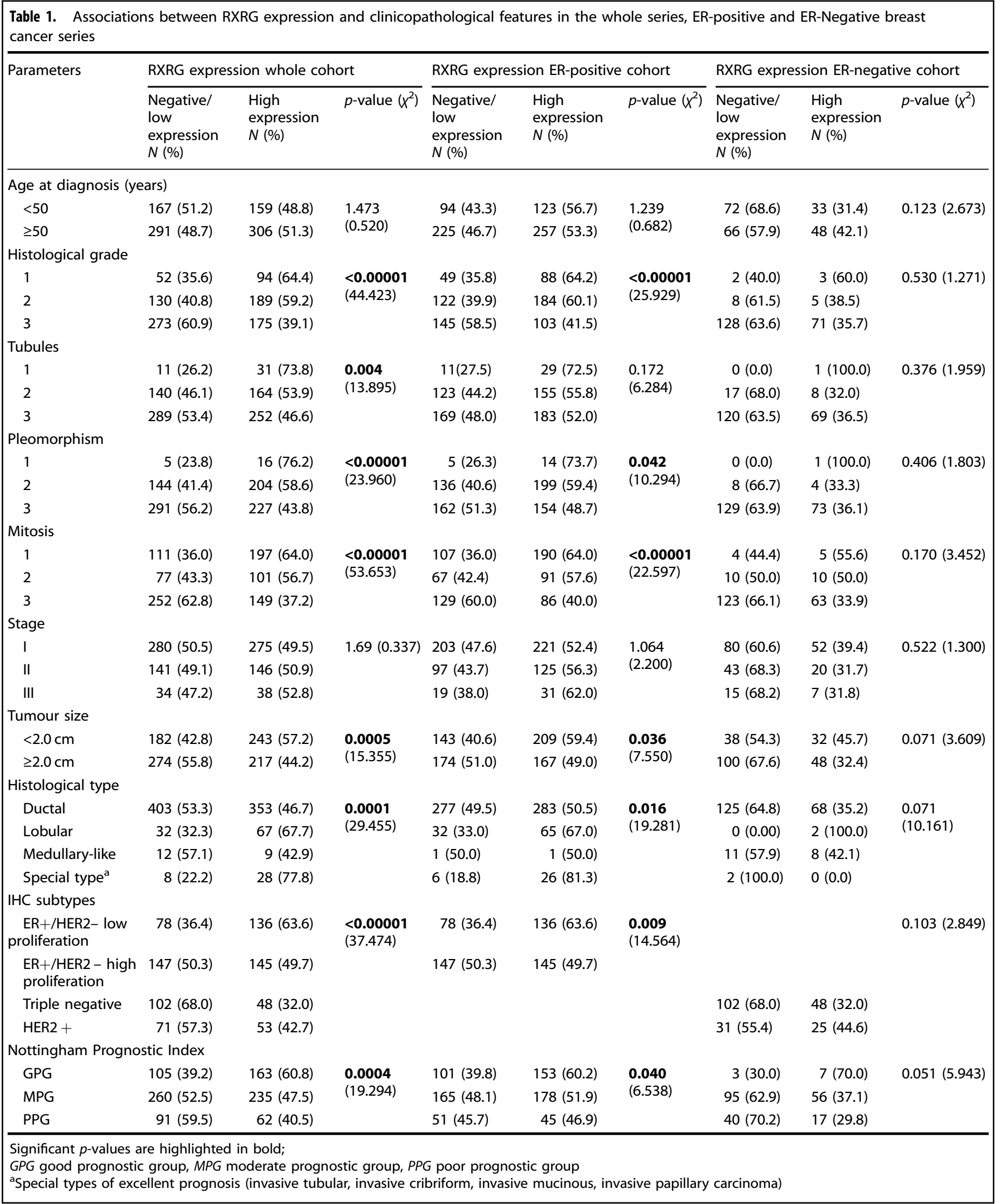

histological grade, nodal stage, ER status and proliferative fraction as assessed by Ki67. Comparable results were obtained when we included the ER level of expression as a continuous variable to the multivariate analysis of the ER-positive cohort (Table 4).
Similarly, in ER-positive tumours, high RXRG levels were predictive of longer breast cancer-specific survival $(p<0.0001$; Fig. 2c) and longer distant metastasis-free interval ( $p=0.002$; Fig. 2d). The Cox regression model demonstrated that RXRG was 
Table 2. Associations between RXRG expression and other biomarkers in the whole series, in ER-positive and ER-negative breast cancer series

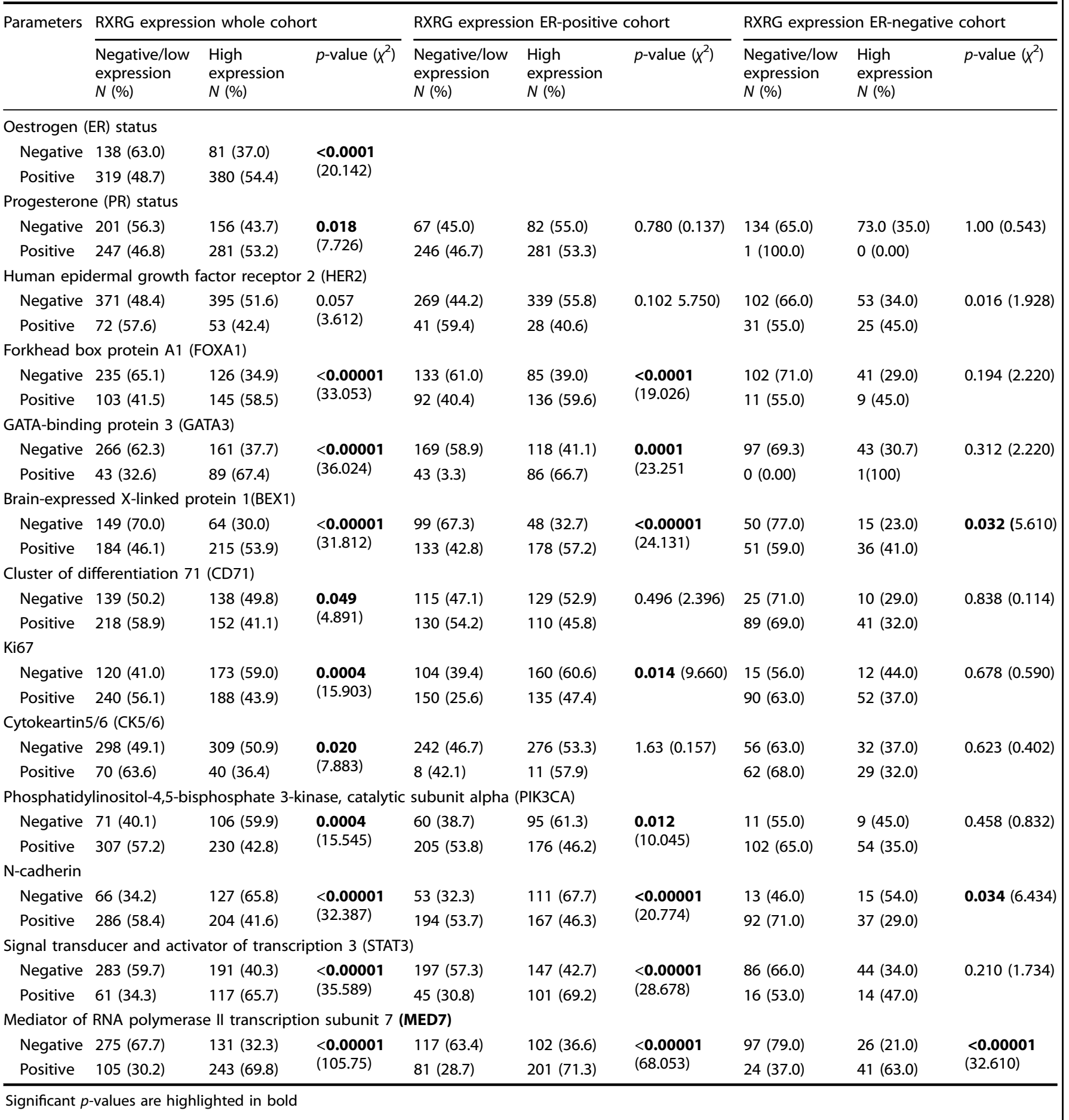

an independent predictor of both breast cancer-specific survival and longer distant metastasis-free interval $(\mathrm{HR}=0.5 ; 95 \% \mathrm{Cl}=$ $0.4-0.7 ; p=0.004$ and $\mathrm{HR}=0.7 ; 95 \% \mathrm{Cl}=0.5-0.9 ; p=0.036$, respectively, Table 4). In triple-negative breast cancer and HER2+ phenotypes, RXRG expression was neither associated with breast cancer-specific survival nor with distant metastasis-free interval.

RXRG positivity was associated with a significant survival advantage in patients with ER-positive tumours irrespective of hormonal therapy $(p=0.049$ and $p<0.0001$, respectively, Fig. 2e, f). Similarly, in ER-positive patients who either received or did not receive adjuvant chemotherapy, the prognostic advantage of positive RXRG expression was maintained $(p=0.006$ and $p=$ 0.002 , respectively) (Fig. $2 g$, h). Supporting this, evaluation of the interaction between RXRG and ER level of expression (RXRG*ER) using the Cox regression model showed significant association with longer breast cancer-specific survival and distant metastasisfree interval (both $p=0.001$ ).

There was a trend towards a positive linear correlation between RXRG mRNA and protein expression in the subset of Nottingham cases within the METABRIC study $(n=150)$, that has data on both 


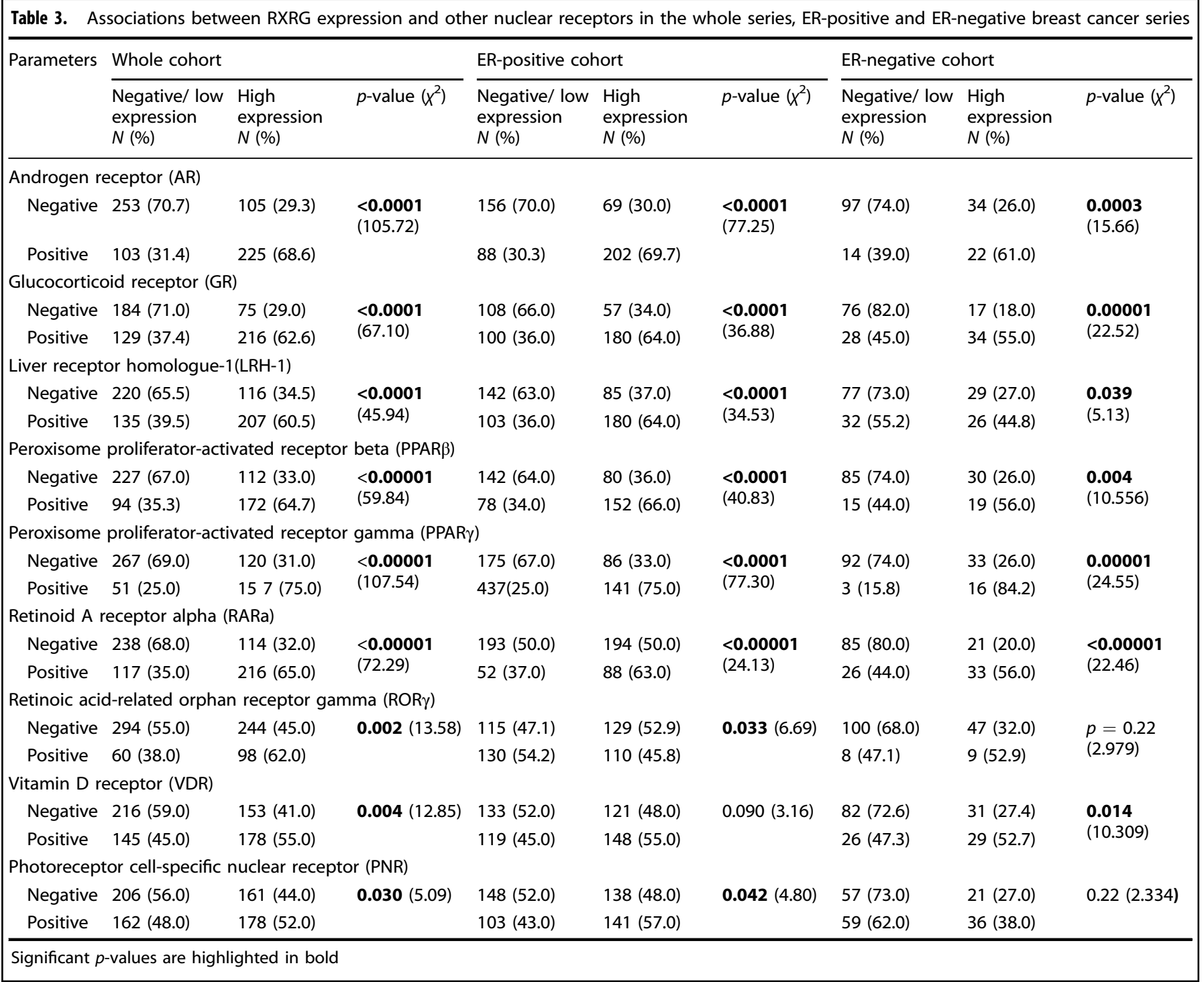

mRNA and protein expression, however, the association did not reach statistical significance $(r=0.20, p=0.077)$.

Genomic study and pathway analysis

We next identified differential gene expression between patients with low versus high RXRG mRNA expression in the Nottingham primary operable breast cancer series, which were included in the METABRIC ${ }^{34}$ study $(n=150)$. This analysis identified 1048 significant differentially expressed genes $(p<$ $0.05)$, comprises of 554 over-expressed and 494 downregulated genes, associated with reduced RXRG expression. Analysis of the differential gene expression list identified over-represented pathways, including dysregulation of genes regulating ER signalling pathway (Supplementary Table 2; $p=0.0053$; FOS and $A P-1$ transcription factor subunit). Other relevant pathways involved in regulating RXRG protein expression included CAMP signalling pathway $(p=0.001 ; A D O R A 1)$, protein digestion and absorption pathway $(p=0.001 ; C O L 4 A 2$ and SLC7A7 and the $A B C$ transport pathway $(p=0.002 ; A B C B 9$ and $A B C D 3)$. Interaction with RXRG and other nuclear receptors in steroid signalling pathways are summarised in Supplementary Fig. 2.
$R X R G$ genomic profiling

Expression analysis for RXRG mRNA using Breast Cancer GeneExpression Miner v4.0 showed that high RXRG expression was associated with older age at diagnosis ( $n=3600$; Supplementary Fig. 3a; $p=0.0082)$, lower histological tumour grade $(n=3518 ; p$ $=0.0024$; Supplementary Fig. $3 \mathrm{~b})$, ER-positive status $(n=5558$; Supplementary Fig. $3 c ; p=0.029$ ). Among PAM50 subtypes, RXRG mRNA was associated with luminal subtypes $(n=5607 ; p=$ 0.0024 ; Supplementary Fig. $3 d$ ) and non-triple-negative status ( $n$ $=1275 ; p=0.014$; Supplementary Fig. 3e). Targeted prognostic analyses for $R X R G$ with nodal status and positive ER status patients ( $n=33$ data sets, 3941 patients) indicated that high gene expression correlated with adverse event-free survival $(\mathrm{HR}=$ $0.88 ; 95 \% \mathrm{Cl}=0.79-0.98 ; p=0.025$; Supplementary Fig. 3f). Consistent with this, Kaplan-Meier analysis ${ }^{38}$ indicates high RXRG expression showed significant survival advantage irrespective of systemic treatment in $(n=3951 ; p<0.0001$; Supplementary Fig. $3 g$ ). To confirm this, we examined the TCGA-BRCA ${ }^{44,45}$ data set and found high RXRG mRNA expression was associated with longer disease-free intervals, post-menopausal status, and differential ER, PR and HER2 expression (Supplementary Fig. 4a-f). 

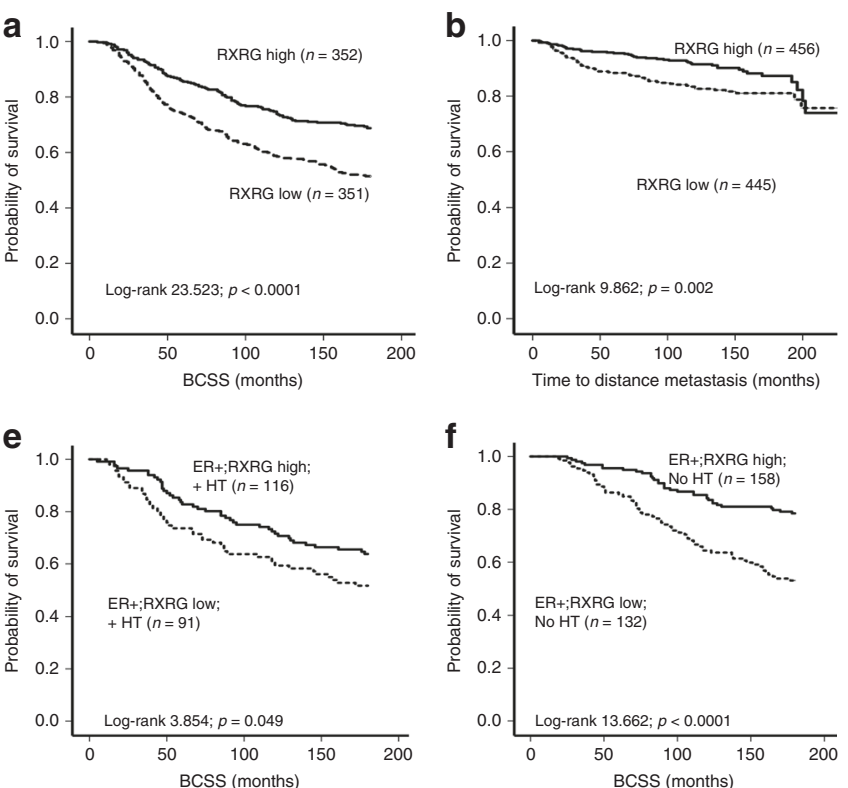
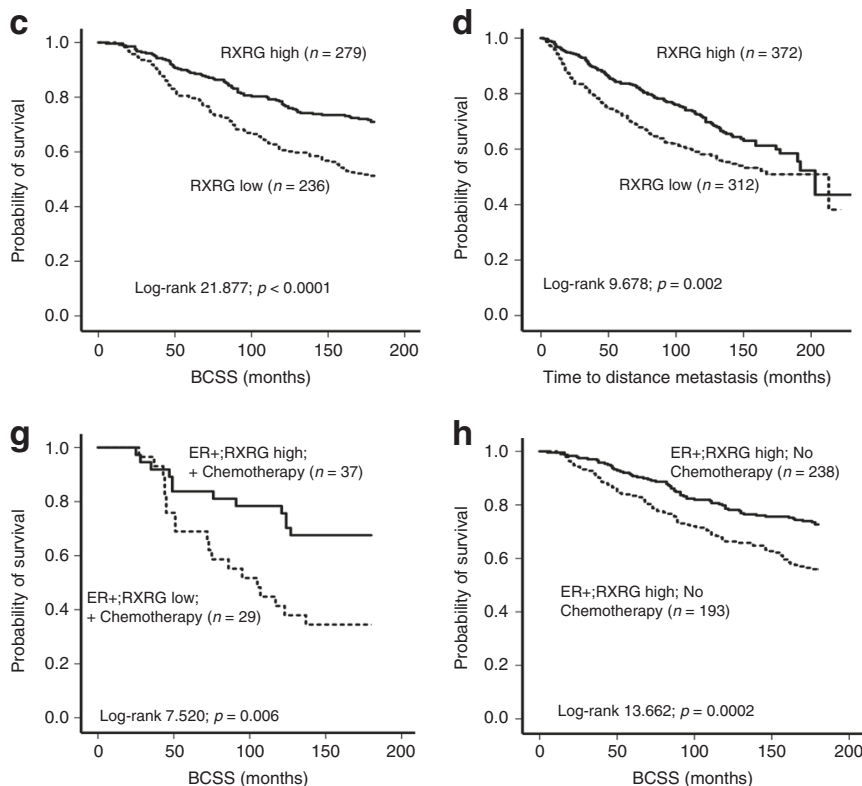

Fig. 2 Kaplan-Meier plot for the association of RXRG nuclear expression. Whole series: a Breast cancer-specific survival, b distant metastasisfree survival. In ER-positive tumours. c Breast cancer-specific survival, $\mathbf{d}$ distant metastasis-free survival. Kaplan-Meier analysis of breast cancerspecific survival showing the impact of treatment on RXRG nuclear protein expression in ER-positive cohort; e in patients who did receive hormone therapy $\mathbf{f}$ in patients that did not receive hormone therapy $\mathbf{g}$ in patients who did receive chemotherapy and $\mathbf{h}$ in patients who did not receive chemotherapy with significance determined using the log-rank test

\section{DISCUSSION}

Understanding the mechanisms by which RXRs exert their effects in breast cancer remains incomplete. ${ }^{12}$ To our knowledge, this is the first study to define the prognostic role RXRG in breast cancer using a large clinical data set with long-term follow up. Results from the current study provide evidence that high expression of RXRG protein was significantly associated good long-term clinical outcome. Our study shows that high-nuclear RXRG was associated with ER-positive tumours, and is consistent with previous reports, which shows it confers a better prognostic impact. ${ }^{46}$ Indeed, the positive correlation between RXRG and ER expression, and association of higher RXRG with improved patient outcome independent of ER expression, suggest that RXRG could be a potential surrogate marker for ER expression in our cohort. Moreover, RXRG expression is significantly higher in breast cancer histologic subtypes with better prognosis such as invasive lobular carcinoma, ${ }^{46,47}$ in contrast to ductal or medullary-like tumours, which typically are associated with poorer outcomes.

In this study, ER-positive breast cancer showed the highest expression of RXRG compared to HER2+ and triple-negative breast cancer. Moreover, elevated expression of RXRG was associated with ER associated markers, such as GATA3, ${ }^{48}$ FOXA $1,{ }^{49}$ BEX, ${ }^{30}$ STAT3 $^{43}$ and MED7. ${ }^{33}$ As noted earlier, RXRs and RARs form heterodimeric complexes, which bind DNA at specific retinoid responsive elements and regulate the various transcriptional processes. ${ }^{12}$ In breast cancer, functional interactions between retinoic acid and oestrogen signalling are complex and well documented. 2,19,20

In this study pathway, analyses were conducted to explore the differentially enriched pathways associated with increased expression levels of RXRG protein. Results on pathway analysis confirmed our IHC findings reinforcing the importance of RXRG expression and ER status, where it revealed a positive association between high RXRG expression and ER positivity, and on patients' survival. Our results indicated that the ER enriched pathway was the top master regulator of RXRG. Thus, we exposed a positive correlation between the genes regulating the ER pathway and RXRG protein expression, suggesting that suppressed expression of those indicators may inhibit signalling via the ER pathway and consequently affecting RXRG expression. For instance, dimerised ER directly binds to DNA sequences called oestrogen response elements (EREs) in relevant activated genes and activate gene transcription. However, ER is also known to use non-classical pathways via Activator protein 1 (AP-1) or via Specificity protein 1 $(\mathrm{Sp}-1){ }^{50}$ In ER-positive, breast cancer cell lines, ER enhanced ADORA1 mRNA and protein levels. Inhibition of ADORA1 reduced the binding activity of ER to its target gene indicating its role for the transcriptional activity of ER on oestrogen stimulation. ${ }^{51}$ By decreasing COL4A2 mRNA levels through miR-29b may be contribute to the tumorigenicity in ER-positive BC cells. ${ }^{52}$ The aforementioned studies have revealed the potential role of these biomarkers in ER-related pathways and may affect RXRG expression. However, it is important to note that the role of RXRG within ER-related pathways may be quite complex, depending on the specific interacting partners. For example, in this study, RXRG expression was negatively associated with PIK3CA. PIK3CA mutations are strongly associated with ER-positive tumours with better prognostic characteristics. ${ }^{53}$ Thus, its inverse relationship to PIK3CA warrants further investigation in the context of ERassociated pathways. Interestingly, in the MNU-induced rat mammary tumour models, the RXR-selective retinoid bexarotene (Targretin), suppressed ER-positive tumour development with minimal toxicity. ${ }^{54}$

In this study, the negative correlation with $\mathrm{N}$-cadherin, $\mathrm{CK} 5 / 6$ and Ki67 indicates that RXRG expression is not associated with aggressive breast cancers. Elevated $\mathrm{N}$-cadherin expression is associated with epithelial-mesenchymal transition (EMT) and tumour aggressiveness. ${ }^{55}$ In thyroid carcinoma, administration of ligands selective for RXRG resulted in a $30 \%$ reduction in cell proliferation, ${ }^{56}$ which is in agreement with low proliferation index and high RXRG expression. High molecular weight cytokeratin are strongly associated with high histological grade, and worse patient outcome $\mathrm{e}^{31}$ and their negative association with RXRG further reinforces its role as a good prognostic indicator.

Nuclear RXRG expression displayed strong positive associations with other nuclear receptors. Studies have shown that RXRs form heterodimers with many nuclear receptors, including RARs, VDRs, PPARs, liver- $X$ receptor (LXRs) and farnesoid $X$ receptors (FXRs), ${ }^{57}$ 
Retinoid X receptor gamma (RXRG) is an independent prognostic biomarker...

\begin{tabular}{|c|c|c|c|c|c|c|c|c|c|c|c|c|}
\hline \multirow[t]{2}{*}{ Variable } & \multicolumn{6}{|c|}{ Breast cancer-specific survival } & \multicolumn{6}{|c|}{ Distant metastasis-free interval } \\
\hline & $\overline{\mathrm{HR}}$ & $95 \% \mathrm{Cl}$ & $p$-value & $\overline{H R}$ & $95 \% \mathrm{Cl}$ & $p$-value & $\overline{\mathrm{HR}}$ & $95 \% \mathrm{Cl}$ & $p$-value & $\overline{\mathrm{HR}}$ & $95 \% \mathrm{Cl}$ & $p$-value \\
\hline \multicolumn{13}{|l|}{ Whole cohort } \\
\hline Tumour size & 2.1 & $1.8-2.5$ & $<0.0001$ & 1.6 & $1.1-2.2$ & 0.006 & 1.9 & $1.6-2.2$ & $<0.0001$ & 1.4 & $1.1-1.9$ & 0.005 \\
\hline$E R^{a}$ & 0.9 & $0.9-1.1$ & $<0.0001$ & 1.1 & $0.9-1.2$ & 0.558 & 0.9 & $0.8-1.1$ & $<0.0001$ & 1.6 & $1.1-2.3$ & 0.026 \\
\hline Ki67 & 2.6 & $2.1-3.1$ & $<0.0001$ & 1.5 & $1.1-2.3$ & 0.027 & 2.1 & $1.7-2.5$ & $<0.0001$ & 1.6 & $1.2-2.2$ & 0.004 \\
\hline RXRG & 0.6 & $0.4-0.7$ & $<0.0001$ & 0.6 & $0.4-0.8$ & 0.040 & 0.8 & $0.6-0.9$ & 0.003 & 0.7 & $0.6-0.9$ & 0.025 \\
\hline \multicolumn{13}{|l|}{ ER+cohort } \\
\hline $\mathrm{ER}^{\mathrm{a}}$ & 0.9 & $0.9-1.0$ & 0.101 & 0.9 & $0.8-1.1$ & 0.428 & 1.0 & $0.9-1.2$ & 0.002 & 0.9 & $0.8-1.1$ & 0.456 \\
\hline Ki67 & 2.9 & $2.3-3.7$ & $<0.0001$ & 1.8 & $1.2-2.9$ & 0.005 & 2.4 & $1.9-3.0$ & $<0.0001$ & 1.8 & $1.2-2.6$ & 0.002 \\
\hline RXRG & 0.5 & $0.4-0.7$ & $<0.0001$ & 0.5 & $0.4-0.7$ & 0.004 & 0.7 & $0.5-0.9$ & 0.002 & 0.7 & $0.5-0.9$ & 0.036 \\
\hline
\end{tabular}

suggesting that the positive correlations in our study could be due to heterodimer formation with one or more of these nuclear receptors. For instance, in breast cancer cells treated with ligands specific for PPARy and RXR/RAR, troglitazone and 9-cis-retinoic acid, respectively, a reduction in proliferation was observed, ${ }^{58}$ and low doses of PPARY and RXR ligands also promoted apoptosis. ${ }^{59}$ This suggests that RXRs have an anti-tumorigenic role, potentially through heterodimer formation with PPARy. Treatment of thyroid cancer cells containing both RXRG and PPARY with their ligands resulted in a synergistic increase in apoptotic activity. ${ }^{56}$ This suggests that RXRY-PPARy heterodimer may be present, and that the activation of this heterodimer leads to a synergistic increase in apoptosis. For this reason, we propose that increased expression of RXRG could potentiate heterodimer formation and activation of other nuclear receptors (e.g., VDR, RAR and PPARY) thereby enhancing their anti-tumorigenic functions.

Regarding the association with patient outcome, high-nuclear RXRG expression was associated with improved breast cancerspecific survival and a longer time to distant metastasis in the whole series and in ER-positive breast cancer. However, in other breast cancer subtypes RXRG did not show any association with patient outcome. This might be due to the smaller sample size of ER-negative, HER2 + and triple-negative breast tumours in this cohort. Further investigation of larger cohorts of ER-negative, HER2 +, and triple-negative breast tumours is therefore warranted. Our findings are consistent with previous reports in breast and renal cancer. ${ }^{60,61}$ In our study, these outcome associations were independent of other well-established prognostic variables. Interestingly, increased RXRG expression showed improved outcome regardless of adjuvant hormonal therapy or chemotherapy status. Hence, in chemotherapy-intolerant patients, therapeutic manipulation of RXRG on its own, or in combination with other therapies, may be helpful in improving the existing treatment regimen, particularly as next-generation RXR subtype-selective rexinoids enter clinical testing and use. Furthermore, assessment of RXRG mRNA levels using bc-GenExMiner and TCGA demonstrated that high $R X R G$ mRNA expression is significantly associated with better tumour characteristics and longer event-free survival of breast cancer patients, which corroborates with RXRG protein expression. RARA mRNA expression levels in breast cancer patients treated with hormonal therapy predicted positive outcome, ${ }^{19}$ which is in agreement with our findings.

In summary, high RXRG expression in breast cancer is associated with favourable prognostic parameters and is an independent prognostic factor with prolonged patient survival. The interaction between RXRG, ER, and other nuclear receptors may explain the prognostic effect of RXRG in breast cancer. There is evidence that rexinoids are more effective anti-cancer agents than retinoids in preclinical models and show minimal toxicity. ${ }^{62}$ Therefore, further studies to validate the potential of RXRG as a therapeutic target in breast cancer are therefore warranted.

\section{ACKNOWLEDGEMENTS}

We thank the Nottingham Health Science Biobank and Breast Cancer Now Tissue Bank for the provision of tissue samples.

\section{AUTHOR CONTRIBUTIONS}

C.J. participated in its conception, design, experimentation, analysis, interpretation and manuscript drafting. S.A. conducted the immunohistochemical studies and participated in the analysis and interpretation. M.S.T. helped with pathology review and manuscript drafting; M.A., F.Q.G. and I.A. helped in immune-histochemical analysis and interpretation; M.A., S.K., I.A., M.A.A., S.A., N.P.M., I.O.E. and A.R.G. participated in interpretation and manuscript drafting. E.A.R. conceived and supervised the study, participated in its design, interpretation and analysis, including drafting. All authors contributed to drafting and reviewing the manuscript and approved the submitted and final version.

\section{ADDITIONAL INFORMATION}

Supplementary information is available for this paper at https://doi.org/10.1038/ s41416-019-0589-0.

Competing interests: The authors declare no competing interests.

Ethical approval and consent to participate: All patients included in this study were consented. This work obtained ethics approval by the North West-Greater Manchester Central Research Ethics Committee under the title:Nottingham Health 
Science Biobank (NHSB), reference number 15/NW/0685. We can declare that this study is complying with Helsinki declaration.

Funding: This research received no specific grant from any funding agency in the public, commercial, or not-for-profit sectors.

Data availability: The authors confirm the data that heen used in this work is available on reasonable request.

Note: This work is published under the standard license to publish agreement. After 12 months the work will become freely available and the license terms will switch to a Creative Commons Attribution 4.0 International (CC BY 4.0).

Publisher's note Springer Nature remains neutral with regard to jurisdictional claims in published maps and institutional affiliations.

\section{REFERENCES}

1. Ferlay, J., Soerjomataram, I., Dikshit, R., Eser, S., Mathers, C., Rebelo, M. et al. Cancer incidence and mortality worldwide: sources, methods and major patterns in GLOBOCAN 2012. Int. J. Cancer. 136, E359-E386 (2015).

2. Hua, S., Kittler, R., White, K. P. Genomic antagonism between retinoic acid and estrogen signaling in breast cancer. Cell. 137, 1259-1271 (2009).

3. Ni, M., Chen, Y., Lim, E., Wimberly, H., Bailey, S. T., Imai, Y. et al. Targeting androgen receptor in estrogen receptor-negative breast cancer. Cancer Cell. 20, 119-131 (2011)

4. Muscat, G. E., Eriksson, N. A., Byth, K., Loi, S., Graham, D., Jindal, S. et al. Research resource: nuclear receptors as transcriptome: discriminant and prognostic value in breast cancer. Mol. Endocrinol. 27, 350-365 (2013).

5. Doan, T. B., Graham, J. D. \& Clarke, C. L. Emerging functional roles of nuclear receptors in breast cancer. J. Mol. Endocrinol. 58, R169-R190 (2017).

6. Abduljabbar, R., Negm, O. H., Lai, C. F., Jerjees, D. A., Al-Kaabi, M., Hamed, M. R. et al. Clinical and biological significance of glucocorticoid receptor (GR) expression in breast cancer. Breast Cancer Res. Treat. 150, 335-346 (2015).

7. Goya, L., Maiyar, A. C., Ge, Y. \& Firestone, G. L. Glucocorticoids induce a G1/G0 cell cycle arrest of Con8 rat mammary tumor cells that is synchronously reversed by steroid withdrawal or addition of transforming growth factor-alpha. Mol. Endocrinol. 7, 1121-1132 (1993).

8. Hilborn, E., Gacic, J., Fornander, T., Nordenskjold, B., Stal, O. \& Jansson, A. Androgen receptor expression predicts beneficial tamoxifen response in oestrogen receptor-alpha-negative breast cancer. Br. J. Cancer. 114, 248-255 (2016).

9. Abedin, S. A., Thorne, J. L., Battaglia, S., Maguire, O., Hornung, L. B., Doherty, A. P. et al. Elevated NCOR1 disrupts a network of dietary-sensing nuclear receptors in bladder cancer cells. Carcinogenesis. 30, 449-456 (2009).

10. Battaglia, S., Maguire, O., Thorne, J. L., Hornung, L. B., Doig, C. L., Liu, S. et al. Elevated NCOR1 disrupts PPARalpha/gamma signaling in prostate cancer and forms a targetable epigenetic lesion. Carcinogenesis. 31, 1650-1660 (2010).

11. Long, M. D. \& Campbell, M. J. Pan-cancer analyses of the nuclear receptor superfamily. Nucl. Receptor Res. 2, 101182 (2015).

12. Tang, X. H. \& Gudas, L. J. Retinoids, retinoic acid receptors, and cancer. Annu. Rev. Pathol. 6, 345-364 (2011).

13. Dawson, M. I. \& Xia, Z. The retinoid X receptors and their ligands. Biochim. Biophys. Acta. 1821, 21-56 (2012)

14. Nuclear Receptors Nomenclature C. A unified nomenclature system for the nuclear receptor superfamily. Cell. 97, 161-163 (1999).

15. Conzen, S. D. Minireview: nuclear receptors and breast cancer. Mol. Endocrinol. 22, 2215-2228 (2008).

16. Kalra, R. S. \& Bapat, S. A. Expression proteomics predicts loss of RXR-gamma during progression of epithelial ovarian cancer. PLOS ONE. 8, e70398 (2013).

17. Lee, S. M., Lee, J. Y., Choi, J. E., Lee, S. Y., Park, J. Y. \& Kim, D. S. Epigenetic inactivation of retinoid $X$ receptor genes in non-small cell lung cancer and the relationship with clinicopathologic features. Cancer Genet. Cytogenet. 197, 39-45 (2010).

18. Zanardi, S., Serrano, D., Argusti, A., Barile, M., Puntoni, M. \& Decensi, A. Clinical trials with retinoids for breast cancer chemoprevention. Endocr. Relat. Cancer. 13, 51-68 (2006).

19. Ross-Innes, C. S., Stark, R., Holmes, K. A., Schmidt, D., Spyrou, C., Russell, R. et al. Cooperative interaction between retinoic acid receptor-alpha and estrogen receptor in breast cancer. Genes Dev. 24, 171-182 (2010).

20. Kittler, R., Zhou, J., Hua, S., Ma, L., Liu, Y., Pendleton, E. et al. A comprehensive nuclear receptor network for breast cancer cells. Cell Rep. 3, 538-551 (2013).

21. Abd El-Rehim, D. M., Ball, G., Pinder, S. E., Rakha, E., Paish, C., Robertson, J. F. et al. High-throughput protein expression analysis using tissue microarray technology of a large well-characterised series identifies biologically distinct classes of breast cancer confirming recent cDNA expression analyses. Int. J. Cancer. 116, 340-350 (2005).

22. Aleskandarany, M. A., Green, A. R., Benhasouna, A. A., Barros, F. F., Neal, K., ReisFilho, J. S. et al. Prognostic value of proliferation assay in the luminal, HER2positive, and triple-negative biologic classes of breast cancer. Breast Cancer Res. 14, R3 (2012)

23. Blamey, R. W., Ellis, I. O., Pinder, S. E., Lee, A. H., Macmillan, R. D., Morgan, D. A. et al. Survival of invasive breast cancer according to the Nottingham Prognostic Index in cases diagnosed in 1990-1999. Eur. J. Cancer. 43, 1548-1555 (2007).

24. McCarty, K. S. Jr. \& KS, Mc. Carty Sr. Histochemical approaches to steroid receptor analyses. Semin. Diagn. Pathol. 1, 297-308 (1984).

25. Aleskandarany, M. A., Rakha, E. A., Ahmed, M. A., Powe, D. G., Ellis, I. O. \& Green, A. R. Clinicopathologic and molecular significance of phospho-Akt expression in early invasive breast cancer. Breast Cancer Res. Treat. 127, 407-416 (2011).

26. Rakha, E. A., El-Sayed, M. E., Green, A. R., Lee, A. H., Robertson, J. F. \& Ellis, I. O. Prognostic markers in triple-negative breast cancer. Cancer. 109, 25-32 (2007).

27. Abduljabbar, R., Al-Kaabi, M. M., Negm, O. H., Jerjees, D., Muftah, A. A., Mukherjee, A. et al. Prognostic and biological significance of peroxisome proliferatoractivated receptor-gamma in luminal breast cancer. Breast Cancer Res. Treat. 150, 511-522 (2015).

28. Aleskandarany, M. A., Abduljabbar, R., Ashankyty, I., Elmouna, A., Jerjees, D., Ali, S. et al. Prognostic significance of androgen receptor expression in invasive breast cancer: transcriptomic and protein expression analysis. Breast Cancer Res. Treat. 159, 215-227 (2016).

29. Habashy, H. O., Powe, D. G., Staka, C. M., Rakha, E. A., Ball, G., Green, A. R. et al. Transferrin receptor (CD71) is a marker of poor prognosis in breast cancer and can predict response to tamoxifen. Breast Cancer Res. Treat. 119, 283-293 (2010).

30. Naderi, A., Teschendorff, A. E., Beigel, J., Cariati, M., Ellis, I. O., Brenton, J. D. et al. $B E X 2$ is overexpressed in a subset of primary breast cancers and mediates nerve growth factor/nuclear factor-kappaB inhibition of apoptosis in breast cancer cell lines. Cancer Res. 67, 6725-6736 (2007).

31. Alshareeda, A. T., Soria, D., Garibaldi, J. M., Rakha, E., Nolan, C., Ellis, I. O. et al. Characteristics of basal cytokeratin expression in breast cancer. Breast Cancer Res. Treat. 139, 23-37 (2013).

32. Habashy, H. O., Powe, D. G., Rakha, E. A., Ball, G., Paish, C., Gee, J. et al. Forkheadbox A1 (FOXA1) expression in breast cancer and its prognostic significance. Eur. J. Cancer. 44, 1541-1551 (2008).

33. Joseph, C., Macnamara, O., Craze, M., Russell, R., Provenzano, E., Nolan, C. C. et al. Mediator complex (MED) 7: a biomarker associated with good prognosis in invasive breast cancer, especially ER+ luminal subtypes. Br. J. Cancer 118, 1142-1151 (2018).

34. Rakha, E. A., Martin, S., Lee, A. H., Morgan, D., Pharoah, P. D., Hodi, Z. et al. The prognostic significance of lymphovascular invasion in invasive breast carcinoma. Cancer. 118, 3670-3680 (2012)

35. Jezequel, P., Campone, M., Gouraud, W., Guerin-Charbonnel, C., Leux, C., Ricolleau, G. et al. bc-GenExMiner: an easy-to-use online platform for gene prognostic analyses in breast cancer. Breast Cancer Res. Treat. 131, 765-775 (2012).

36. Kurozumi, S., Joseph, C., Sonbul, S., Gorringe, K. L., Pigera, M., Aleskandarany, M. A. et al. Clinical and biological roles of Kelch-like family member 7 in breast cancer: a marker of poor prognosis. Breast Cancer Res. Treat. 170, 525-533 (2018).

37. Cancer Genome Atlas N. Comprehensive molecular portraits of human breast tumours. Nature. 490, 61-70 (2012).

38. Gyorffy, B., Lanczky, A., Eklund, A. C., Denkert, C., Budczies, J., Li, Q. et al. An online survival analysis tool to rapidly assess the effect of 22,277 genes on breast cancer prognosis using microarray data of 1,809 patients. Breast Cancer Res. Treat. 123, 725-731 (2010).

39. Lohse, M., Bolger, A. M., Nagel, A., Fernie, A. R., Lunn, J. E., Stitt, M. et al. RobiNA: a user-friendly, integrated software solution for RNA-Seq-based transcriptomics. Nucl. Acids Res. 40, W622-W627 (2012).

40. Zhang, B., Kirov, S. \& Snoddy, J. WebGestalt: an integrated system for exploring gene sets in various biological contexts. Nucl. Acids Res. 33, W741-W748 (2005).

41. Camp, R. L., Dolled-Filhart, M. \& Rimm, D. L. X-tile: a new bio-informatics tool for biomarker assessment and outcome-based cut-point optimization. Clin. Cancer Res. 10, 7252-7259 (2004)

42. Szklarczyk, D., Franceschini, A., Wyder, S., Forslund, K., Heller, D., Huerta-Cepas, J. et al. STRING v10: protein-protein interaction networks, integrated over the tree of life. Nucl. Acids Res. 43, D447-D452 (2015).

43. Aleskandarany, M. A., Agarwal, D., Negm, O. H., Ball, G., Elmouna, A., Ashankyty, I. et al. The prognostic significance of STAT3 in invasive breast cancer: analysis of protein and mRNA expressions in large cohorts. Breast Cancer Res. Treat. 156, 9-20 (2016).

44. Cerami, E., Gao, J., Dogrusoz, U., Gross, B. E., Sumer, S. O., Aksoy, B. A. et al. The cBio cancer genomics portal: an open platform for exploring multidimensional cancer genomics data. Cancer Discov. 2, 401-404 (2012). 
45. Gao, J., Aksoy, B. A., Dogrusoz, U., Dresdner, G., Gross, B., Sumer, S. O. et al. Integrative analysis of complex cancer genomics and clinical profiles using the cBioPortal. Sci. Signal. 6, pl1 (2013).

46. Hennigs, A., Riedel, F., Gondos, A., Sinn, P., Schirmacher, P., Marme, F. et al. Prognosis of breast cancer molecular subtypes in routine clinical care: a large prospective cohort study. BMC Cancer. 16, 734 (2016).

47. Rakha, E. A., El-Sayed, M. E., Menon, S., Green, A. R., Lee, A. H. \& Ellis, I. O. Histologic grading is an independent prognostic factor in invasive lobular carcinoma of the breast. Breast Cancer Res. Treat. 111, 121-127 (2008).

48. Hosoda, M., Yamamoto, M., Nakano, K., Hatanaka, K. C., Takakuwa, E., Hatanaka, Y. et al. Differential expression of progesterone receptor, FOXA1, GATA3, and p53 between pre- and postmenopausal women with estrogen receptor-positive breast cancer. Breast Cancer Res. Treat. 144, 249-261 (2014).

49. Hurtado, A., Holmes, K. A., Ross-Innes, C. S., Schmidt, D. \& Carroll, J. S. FOXA1 is a key determinant of estrogen receptor function and endocrine response. Nat. Genet. 43, 27-33 (2011)

50. Safe, S. \& Kim, K. Non-classical genomic estrogen receptor (ER)/specificity protein and ER/activating protein-1 signaling pathways. J. Mol. Endocrinol. 41, 263-275 (2008).

51. Lin, Z., Yin, P., Reierstad, S., O'Halloran, M., Coon, V. J., Pearson, E. K. et al. Adenosine $\mathrm{A} 1$ receptor, a target and regulator of estrogen receptoralpha action, mediates the proliferative effects of estradiol in breast cancer. Oncogene. 29, 1114-1122 (2010).

52. Wang, C., Gao, C., Zhuang, J. L., Ding, C. \& Wang, Y. A combined approach identifies three mRNAs that are down-regulated by microRNA-29b and promote invasion ability in the breast cancer cell line MCF-7. J. Cancer Res. Clin. Oncol. 138 2127-2136 (2012).

53. Dumont, A. G., Dumont, S. N. \& Trent, J. C. The favorable impact of PIK3CA mutations on survival: an analysis of 2587 patients with breast cancer. Chin. J. Cancer. 31, 327-334 (2012).
54. Gottardis, M. M., Bischoff, E. D., Shirley, M. A., Wagoner, M. A., Lamph, W. W. \& Heyman, R. A. Chemoprevention of mammary carcinoma by LGD1069 (Targretin): an RXR-selective ligand. Cancer Res. 56, 5566-5570 (1996).

55. Hulit, J., Suyama, K., Chung, S., Keren, R., Agiostratidou, G., Shan, W. et al. N cadherin signaling potentiates mammary tumor metastasis via enhanced extracellular signal-regulated kinase activation. Cancer Res. 67, 3106-3116 (2007).

56. Klopper, J. P., Hays, W. R., Sharma, V., Baumbusch, M. A., Hershman, J. M. \& Haugen, B. R. Retinoid X receptor-gamma and peroxisome proliferator-activated receptor-gamma expression predicts thyroid carcinoma cell response to retinoid and thiazolidinedione treatment. Mol. Cancer Ther. 3, 1011-1020 (2004).

57. Shulman, A. I. \& Mangelsdorf, D. J. Retinoid $x$ receptor heterodimers in the metabolic syndrome. N. Engl. J. Med. 353, 604-615 (2005).

58. Elstner, E., Williamson, E. A., Zang, C., Fritz, J., Heber, D., Fenner, M. et al. Novel therapeutic approach: ligands for PPARgamma and retinoid receptors induce apoptosis in bcl-2-positive human breast cancer cells. Breast Cancer Res. Treat. 74, 155-165 (2002)

59. Bonofiglio, D., Cione, E., Qi, H., Pingitore, A., Perri, M., Catalano, S. et al Combined low doses of PPARgamma and RXR ligands trigger an intrinsic apoptotic pathway in human breast cancer cells. Am. J. Pathol. 175, 1270-1280 (2009).

60. Heublein, S., Mayr, D., Meindl, A., Kircher, A., Jeschke, U. \& Ditsch, N. Vitamin D receptor, Retinoid $\mathrm{X}$ receptor and peroxisome proliferator-activated receptor gamma are overexpressed in BRCA1 mutated breast cancer and predict prognosis. J. Exp. Clin. Cancer Res. 36, 57 (2017).

61. Obara, W., Konda, R., Akasaka, S., Nakamura, S., Sugawara, A. \& Fujioka, T. Prognostic significance of vitamin $D$ receptor and retinoid $X$ receptor expression in renal cell carcinoma. J. Urol. 178, 1497-1503 (2007).

62. Uray, I. P., Dmitrovsky, E. \& Brown, P. H. Retinoids and rexinoids in cancer prevention: from laboratory to clinic. Semin. Oncol. 43, 49-64 (2016). 